\title{
How the twins do it: STR and the clock paradox
}

\section{Graham Nerlich}

\section{Introduction}

In their interesting and controversial paper ${ }^{1}$ McCall and Lowe (2003) (hereafter 'the authors') claim that the notorious twins (or clock) paradox in Special Relativity (hereafter STR) may be understood not only in the context of 4D geometry but also, and equally well, in a 3D setting. They claim that 3D and 4D descriptions are equivalent in the sense of being intertranslatable within STR $(\mathbb{S} 4)$. Their $3 \mathrm{D}$ analysis aims to solve the twins paradox through the use of A-theory concepts and, in particular, by using the idea that time flows at different rates with respect to different frames of reference (115).

I will assume (as the authors do) the thesis of $3 \mathrm{D} / 4 \mathrm{D}$ equivalence in the sense that $3 \mathrm{D}$ and $4 \mathrm{D}$ descriptions are intertranslatable. Under that assumption, I study the paradox in terms of 3D/B-theoretic concepts, a style left untouched by the authors. My solution calls on the proper times of the twins (or clocks) and makes no use of different frames of reference. This implies that neither the A-theory nor alternative frames play a necessary role.

\section{What is the twins paradox?}

The twins or clock paradox is a well-known consequence of STR. It is a paradox only in that it clashes strongly with the intuitions we have about time before we are at home with the theory. Although the paradox is familiar, I will sketch it and its 4D solution.

Let there be two ideal ${ }^{2}$ clocks which both read 0 at some point-event $\mathrm{P}$. Thus they agree in reading 0 together at the same time and place and so they are absolutely synchronized. After various vicissitudes the clocks meet

\footnotetext{
${ }^{1}$ Numerals in round brackets refer to pages in this paper.

2 STR admits the concept of an ideal clock, one on which acceleration has no effect. That is the clock bypothesis. See Rindler 1977: 43.
} 
again at some later point-event $\mathrm{Q}$ and their readings are compared again at that place and time (hence, again, the comparison is absolute). In general, the readings on the clocks at $\mathrm{Q}$ will disagree. We may add colour to the example by substituting for the clocks a pair of twins. ${ }^{3}$ The twins (Jack and Jill) leave $\mathrm{P}$ at the same age, but when they meet at $\mathrm{Q}$ one will be younger than the other (in general), depending on how they have journeyed.

A simple case is often used to illustrate the general claim. Jack remains at rest in an inertial frame throughout and his sister Jill moves straight out and straight back (far and fast, let's suppose) on her journey. Let Jack's inertial frame be the one with respect to which $\mathrm{P}$ and $\mathrm{Q}$ occur at the same place but at different times. Jack's age (hence his clock's time) is a (maximal) limit of all the ages (times) of twins and clocks that are or could be at both $\mathrm{P}$ and Q.

The authors' 4D explanation uses a sketch of the space-time trajectories of Jack and Jill in the simple case. (See Figure 1.) In fact, the figure represents something simpler still, the case of three inertial clocks, two of which closely approximate Jill's space-time trajectory. Their temporal lengths also closely approximate the interval measured by Jill's clock. Although the figure makes an angle at $Y$ rather than a curve when Jill accelerates to start back again, what happens just in that period is largely irrelevant to explaining the different ages of the twins, as the authors agree (119). ${ }^{4}$ The diagram

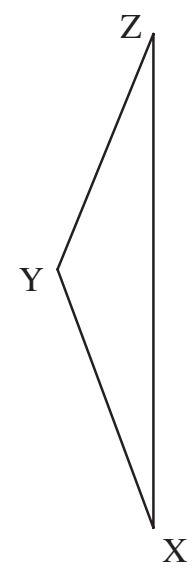

Figure 1

${ }^{3}$ Clocks provide the advantage of letting us toy with fine numerical measures of time in describing the paradox.

${ }^{4}$ Of course it is crucial to the simple case that Jack's world line is a straight line and Jill's is not. 
is particularly vivid and intuitive, since we see at once that the twins' journeys are not symmetrical in space-time: the direct path from $\mathrm{X}$ to $\mathrm{Z}$ does not equal the dogleg path that goes via $\mathrm{Y}^{5}$ Thus the clock that goes from $\mathrm{X}$ to $\mathrm{Z}$ directly, measures a different space-time interval than the one that goes via Y. It does not measure it in a different way or by a clock which runs at a different rate. ${ }^{6}$

In the general (rather than merely the simple) case, neither Jack nor Jill need remain at rest at any stage in any frame of reference. Their journeys are arbitrary timelike curves from $\mathrm{P}$ to $\mathrm{Q}$. Unless their journeys are the same temporal length from $\mathrm{P}$ and $\mathrm{Q}$, their ages differ at $\mathrm{Q}$.

\section{3D description and the A-theory}

The 4D explanation makes no use of coordinate systems or frames of reference. The age difference is frame invariant, as the authors agree (115, 121). It is not evident why translating the explanation into 3D language should - or how it could - exploit different frames of reference. Of course a 3D description obliges us to think of space and time as somehow separated, but why do we need different frames of reference to explain what we explained before without them?

The authors do not discuss what rules of translation underlie the claim that $3 \mathrm{D}$ and $4 \mathrm{D}$ descriptions are equivalent (in the sense of intertranslatable) within STR. My translation of the 4D picture of the simple case is different from theirs, so I must defend it. But otherwise I won't consider what they say in introducing the A-theory into the context of the twins.

The authors translate 'temporal length' in the 4D description of the simple case by the words 'process' and 'rate' (121). They claim that these words are conceptually bound to 3D language, and, more contentiously, bound to the much richer A-theory. I doubt both claims, but consider the second. I note, first, that $3 \mathrm{D}$ description is not necessarily A-theoretic. Further, a 3D/B-theoretic definition may be given of 'process' and 'rate': a process is a set of states of some continuant, differing at different (earlier or later) times; a rate is a measure of how many changes per unit time occur in a process. Nothing in this implies a real (i.e. not merely indexical) present or a flow of time. Finally, a translation of temporal length (as a 4D concept) into temporal rate as a 3D one (121) is neither apt nor necessary. Temporal distance in 4D language and 'process' and 'rate' in 3D language are not 'two sides of the same coin' (121) because neither of the latter terms refers, as 'temporal distance' does, to quantities of time.

5 The Euclidean diagram misrepresents the Minkowski geometry in one respect; longer Euclidean lines correspond to shorter temporal lengths in Minkowski's metric.

6 Taylor and Wheeler (1992: 76-77) are emphatic about this. 
Instead, 'temporal length' and 'temporally long' translate just into 'long time'. To say that one journey takes a longer time than another is the commonest of common (and hence 3D) language. Of course, in saying that, we usually assume that the journeys either began at different times or ended at different ones. But in the paradox, where the journeys begin at the same time and end at the same time, the phrase tells the story accurately. That is exactly what jolts our intuitions.

This does little to explain the paradox. It just restates it in a particular, accurate way.

Clocks don't measure 'rates of elapsed time' or measure 'rates of temporal flow' (121). They measure time: what time it is and that it's a long time since lunch. 'Rates of time' is unclear, calling for special justifying argument and explication.

\section{A 3D explanation of the paradox}

The paradox is not unique to STR. Lorentz $(1904, \mathbb{} \$ 4)$ deduced a virtual equivalent of the slowing of a moving clock. Its spatial counterpart, the Fitzgerald length contraction, was also prominent in the literature before 1905. The idea of frames of reference is alien to these early findings. Let me begin at something of a tangent, then, and deduce the twins paradox in a simplified (ether free) Lorentzian absolute space and time theory. It is easy. Then we need to concern ourselves with only the metaphysical differences between this theory and STR, taking the physics to be the same. ${ }^{7}$ Simplified Lorentz theory has attracted a number of philosophers (e.g. Prior, Tooley, Craig).

In this theory, as in STR, it is a fundamental principle that the $\mathrm{c}$ in Maxwell's equations for the electromagnetic field is a finite constant and has the dimensions of a speed. Let us assume that this is the speed of light and electromagnetic radiation. ${ }^{8}$ This means that we can construct an excellent clock from a couple of mirrors at a fixed distance from, and parallel to, one another. Between them a photon bounces and a counter counts how many bounces strike one of the mirrors. If we take the constant, finite speed of light as fundamental to the theory then it is fundamental that this makes a good clock.

Now suppose that Jack is at absolute rest, so that his clock is the gold standard of absolute time. That is, the bouncing photon that drives his clock moves between the mirrors along the same path up and down. Jill

${ }^{7}$ A dangerous phrase in some contexts, no doubt, but not misleading in this one. Lorentz theory is just STR with an arbitrary frame chosen to found absolute rest.

${ }^{8}$ The assumption could fail if, for instance, the photon is massive. But we may ignore that. 


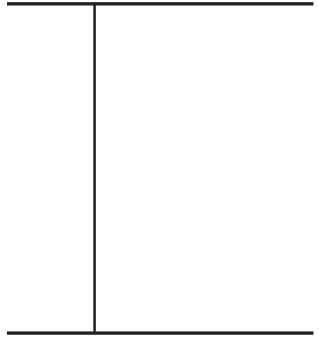

At rest

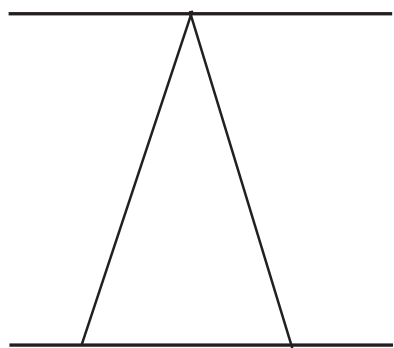

In motion

Figure 2

moves off in a straight line at uniform speed, taking her clock with her. For simplicity, suppose that the line of her motion lies in the plane of one of the mirrors, so that it is orthogonal to the direction of the bouncing photon which works Jack's clock. Then her clock will run slow because the photon will not move up and down along the same track orthogonal to the planes of the mirrors, but along different and longer paths at angles to her mirrors (Figure 2). So her photon bounces less often and her clock's counter will tick less often than Jack's. This has nothing to do with how Jack measures Jill's clock. The paths her photon takes are absolutely longer than the path along which Jack's photon travels. The slowing is absolute. Jill's clock simply doesn't measure The Time.

Clearly enough, if Jill reverses the direction of her journey and returns to Jack, the photon working her clock will still move along slanting paths between her mirrors, so that when she returns, her clock will have ticked less often than Jack's. That will also be true when she accelerates. The only way the path can be minimal (up and down along the same track) and the clock tick properly, is if it is at absolute rest.

Most kinds of clocks involve electromagnetic principles (which underlie the constitution of matter as we ordinarily encounter it), so we can extend the light clock finding to electromagnetic behaviour broadly and thus to ageing processes at large. Jill's wrinkles (and her thought processes) involve them, too. So she will age less than Jack in the same absolute time.

In 3D/B-theoretic language, that explains why Jill is younger than Jack. Her clock and her wrinkles don't reflect The Time. She has aged at a different rate. She has lived through the same period of time, but at less cost in ageing.

\section{Frames of reference}

As a relativity theory, STR tells a different story which departs from the absolutist one just in its metaphysics. That is what we must now explore. 
If we use 3D concepts in STR, then we need ways to separate space and time. If we also want a global story within which we can compare and measure everything in one perspective, then we need a frame of reference. We need one to embrace both Jack and far-off-Jill in a single view. That entails that we will use coordinate time, the global time of the frame we choose. But coordinate time is not the time that matters: proper time matters. I pursue that theme shortly.

But first, there is an obvious frame for the global coordinate story: the one in which $\mathrm{P}$ and $\mathrm{Q}$ happen in the same place. In the simple case, that is also the frame in which Jack is at rest. Choose that frame and the global story is just what it is in Lorentz space-time, save that the photon path lengths are defined relative to the frame, not absolutely.

We could choose another frame and the story would change in respect of path lengths relative to the new frame, and so would coordinate parts of the description. But not the ages, the age difference, and how many times each clock ticked. They are not coordinate quantities, but proper ones invariant, as the authors agree. Those quantities are the ones that matter and coordinate times do not, in general, bear directly on them.

Proper quantities emerge if we separate time from space locally for each twin. There is a metaphysical reason for doing this.

Lorentz absolute space and time allow us to define ontologically a state of absolute rest, but there are two problems with it. The first is epistemological and long familiar: there is no way to tell when anything is at absolute rest. Measuring instruments conspire to hide the difference between rest and uniform, force-free motion.

Worse follows, although it is seldom mentioned. ${ }^{9}$ We have virtually no access to intrinsic physical properties. Let mass be our example. In the Lorentz world the absolute mass of a moving thing increases as a function of its speed, even when speed is uniform and force free. Mass is an intrinsic quantity, but we can never tell what it is. When we measure the mass of something moving absolutely with us, we get its rest mass, not its real intrinsic mass. When at rest, we measure its intrinsic mass correctly, but have no way of knowing that we do. The ontological cost is that intrinsic properties change with mere motion; the epistemic loss is that the world conceals them. Since we can define all classical physical properties (except for charge) in terms of mass, length and time, the epistemic barrier between us and the real world of intrinsic properties is virtually complete.

Thus the Lorentz absolute theory admits inaccessible, intrinsic, inconstant properties which change without the action of causes.

${ }^{9}$ In fact I know of no discussion of the points that follow explicitly as metaphysical ones, although plenty in relativity texts pretty directly implies it. 
STR sheds these problems: it can allow proper quantities. These correspond to the Lorentzian inaccessible, intrinsic properties which are inconstant without being caused to change. But now they are invariant, accessible properties and inconstant only when caused to change. A thing may change its mass because it is heated, but how could the relative motion of something else change its mass?

In STR, if we separate space and time in a local, natural way, without using global frames, then intrinsic properties are accessible. It is only in global, coordinate perspectives that quantities vary. In itself, a clock tells the right time, its own time, its proper time. Jill's does so even when Jack says that it accelerates. Jill's clock does just what Jack's clock does: it counts the bounces of the photon between its mirrors and, as she encounters it, the photon's path is straight up and back. ${ }^{10}$ Nothing happens to Jill's clock: its intrinsic, proper rate is the same as Jack's. So Jack takes longer to get from P to Q than Jill takes. Jill is as young as she looks, since she has lived a shorter time than Jack. The proper times are the real times.

Proper quantities in general - proper time, proper mass, proper length and the others - are intrinsic. Proper time, conventionally written ' $\tau$ ', is a clock's own time, proper mass an object's own mass. ${ }^{11}$ They are proper not to inertial frames but local to concrete objects and processes themselves. They are invariants, but not because the Lorentz transformation juggles them out unchanged. Their invariance is a demand on the transformation because they are the natural intrinsic properties of $3 \mathrm{D}$ things once we abandon absolute space and time. Proper mass is identical with rest mass, but we do not need the global frame in which the thing is at rest to define or speak of it. On the contrary. Only if the proper mass - the intrinsic mass - is an invariant property of the body can the global perspective work: the relativistic mass, $m_{i}$, is a function just of proper mass, $m_{0}$, and of the frame velocity, $\mathbf{v}$, relative to a frame, $\mathrm{F}_{i}$. So for our target quantity, proper time. The proper time, $\tau$, of an ideal clock (or the proper ageing of a twin) allows the relativistic (coordinate) time, $t_{i}$, to be a function just of $\tau$ and the velocity of the clock or twin relative to a frame, $\mathrm{F}_{i}$. Proper time is the fundamental concept.

In the simple case, in Figure 1, Jack and Jill each begin in free fall (or free float). When Jill's clock ticks 10 times, then the proper time from $\mathrm{X}$ to that

10 This holds even while Jill accelerates, so long as her clock is small; that is, so long as the curvature of the photon path and the difference in length between the upward and downward paths is too slight to be of significance. She will need to adjust her counter to compensate for the shrinking size, however.

${ }^{11}$ Proper mass may be intrinsic to a dynamical system of objects, or to an object qua system. It is not as simple as this suggests. I do not pursue that theme here. But see Taylor and Wheeler 1992: 224-28. 
event is 10 units. But that event is not local to the event at which Jack's clock ticks 10 . The only way to relate those events is through the global perspective of some frame, and different frames relate them differently. Nevertheless, all agree on the proper times from X - 10 units in each case. What makes the later meeting of the twins significant for a comparison of the real, proper times of each is that the local comparisons obviate the need for any frame comparison. Then we see that Jack's journey took longer than Jill's - that the proper, true, intrinsic times are different. Frames have no role to play in it.

If that is right, then the twins don't do it by having different rates of clocks in different frames, let alone different rates of the flow of time in different frames. They do it by taking different journeys, one taking longer than the other.

The rates of clocks can be meaningfully compared when both measure the same time interval, as the twins' clocks do in the Lorentz setting. In relativity, the twins' clocks do not measure the same interval. We can indeed speak of the interval from $\mathrm{P}$ to $\mathrm{Q}$, the length of the timelike line from $\mathrm{P}$ to Q. But neither Jack nor Jill measures this interval, save for Jack in the special case. Comparison of their clock rates makes no sense.

Spatial journeys with the same departure and arrival points generally differ in length. Odometers can measure such lengths directly. Plainly, nothing about the way odometers measure spatial journeys makes sense of why the tyres on Jack's car have worn more than those on Jill's. Simply, Jack's journey is longer. Analogously, Jack is more wrinkled than Jill because his timelike journey is longer.

In the simple case Jack's proper time is also the coordinate time of his rest frame. But we can't go on to consider Jill's coordinate time. She has no global frame. (Rather, the obvious ways she might try to take a global view yield contradictions.) In the general case, neither twin has a natural global perspective and comparing their journeys globally, step by step, is much easier from the perspective of an inertial frame, like the obvious one discussed before. Nothing is gained by changing frames.

The frame-relative 'slowing of a moving clock' is a measured, indirect slowing (Resnick 1968: 63, 203; Rindler 1977: 28-29, 43-44; Taylor and Wheeler: 76-77), relative to the frame's global coordinate time. It involves, say, checking the 'moving' clock's rate against a series of clocks synchronized according to the frame; it is mediated by the frame's relation of simultaneity. It is not about Jill's clock in itself.

Further, emphasizing frame-relative differences may well confuse. In frame-relative or coordinate time, Jack, in the simple case, measures the minimum of all coordinate times from $\mathrm{P}$ to $\mathrm{Q}$, since he is at rest in the frame in which they occur in the same place. So for any other frame, the coordinate time interval between $\mathrm{P}$ and $\mathrm{Q}$ is greater. At first glance, the discrep- 
ancy runs in the direction opposite to the one needed to explain the different ages. This does confuse people. ${ }^{12}$ In Figure 1, Jill's journey is approximated by a pair of inertial frames; each of them measures Jack's clock as running slow in the approximating period. That is because each of their global perspectives includes only Jack's early part (for one frame) or his late part (for the other) and much of what happens to his clock is measured by the frames in periods which fall outside the approximation to Jill's trip.

Any frame's measures of Jill are perforce global and not so much wrong as indirect and askew. In 4D language, Jill's cross sections are measured accurately, but on the wrong angle, from the wrong perspective. Although there is nothing subjective in this, it misrepresents her intrinsic nature.

If we stress proper time, proper length and proper mass, then we may rewrite a central message of relativity theory (both special and general) in 3D language in a metaphysically interesting way: every object has its own intrinsic nature, but there is no one standpoint from which all objects may be simply measured as having these properties. In that way, the theory no longer seems to tell the puzzling message that everything is relative, that there is no way things are what they are in themselves. Quite the contrary. But, nevertheless, the world does not present all this together in any single perspective. What the relativity principles then tell us is which global perspectives make sense, if any do. That is a simple message only in STR: inertial frames make sense.

\author{
University of Adelaide \\ Adelaide, South Australia 5005 \\ graham.nerlich@adelaide.edu.au
}

\title{
References
}

Craig, W. L. 2001. Time and the Metaphysics of Reality. Dordrecht: Kluwer.

Lorentz, H. A. 1904. Electromagnetic phenomena in a system moving with any velocity less than that of light. In H. A. Lorentz et al. The Principle of Relativity: a collection of original memoirs on the special and general theory of relativity. 1923. NewYork: Dover.

McCall, S. and E. J. Lowe. 2003. 3D/4D equivalence, the twins paradox and absolute time. Analysis 63: 114-23.

Prior, A. 1970. The notion of the present. Studium Generale 23: 245-48.

Resnick, R. 1968. Introduction to Special Relativity. N.Y.: Wiley.

Rindler, W. 1977. Essential Relativity: Special, General and Cosmological. NewYork: Springer-Verlag.

Taylor, E. F. and J. Wheeler. 1992. Spacetime Physics. N.Y.: Freeman.

Tooley, M. 1997. Time, Tense and Causation. Oxford: Oxford University Press.

12 Taylor and Wheeler 1992: 155-56. They explicitly address it as a source of misunderstanding at the foot of 155 . 